\title{
Fisioterapia aquática no desenvolvimento motor grosso de lactente prematuro com malformações congênitas de membros superiores: relato de caso
}

\author{
KAITIANA MARTINS DA SILVA \\ Associação de Assistência à Criança Deficiente (AACD), São Paulo, SP, Brasil. \\ E-mail: kaitianafisio@hotmail.com \\ CARLA BORGES FLEURI GILL \\ Associação de Assistência à Criança Deficiente (AACD), São Paulo, SP, Brasil. \\ E-mail: carla_bfb@hotmail.com \\ DOUGLAS MARTINS BRAGA \\ Associação de Assistência à Criança Deficiente (AACD), São Paulo, SP, Brasil. \\ E-mail: douglasbraga78@hotmail.com
}

\section{Resumo}

As malformações congênitas e a prematuridade são fatores de risco para o desenvolvimento neuropsicomotor. Sabe-se que os primeiros anos de vida são caracterizados por importantes aquisições do desenvolvimento infantil. Esse período é considerado crucial por causa da maior plasticidade cerebral, com aumento das redes neurais e consequente potencialização dos ganhos de desenvolvimento. Diante dessas características, surge a importância da intervenção o mais precoce possível. O objetivo deste estudo foi verificar a influência da fisioterapia aquática funcional no desenvolvimento motor grosso de um lactente prematuro com diagnóstico clínico de malformação dos ossos do carpo (bilateral). Trata-se de um relato de caso desenvolvido no setor de fisioterapia aquática da Associação de Assistência à Criança Deficiente (AACD) - unidade Ibirapuera. Participou deste estudo um paciente do sexo masculino com 5 meses e 2 dias de idade cronológica, com diagnóstico médico de malformação dos ossos do carpo bilateral e atraso no desenvolvimento neuropsicomotor. Foram realizadas avaliações, antes e depois de oito sessões de atendimento, por meio da Alberta Infant Motor Scale (Aims). O protocolo foi definido com enfoque no estímulo do desenvolvimento neuropsicomotor. Observaram-se aumento de 12 pontos no 
escore bruto da Aims, de 6 a 18, e melhora no percentil. Antes da intervenção, verificou-se percentil 5 e, depois, percentil 50. Este estudo demonstra que, para esse paciente, a fisioterapia aquática funcional pode influenciar de forma positiva o desenvolvimento motor grosso.

\section{Palavras-chave}

Hidroterapia. Desenvolvimento infantil. Prematuridade. Intervenção precoce. Anormalidades congênitas.

\section{INTRODUÇÃO}

Os avanços nas tecnologias de suporte e cuidados aos recém-nascidos pré-termo nas últimas décadas permitiram o aumento da sobrevida dessa população. A Organização Mundial da Saúde (OMS) define como prematuro o recém-nascido com menos de 37 semanas de gestação, independentemente do peso (WORLD HEALTH ORGANIZATION, 2018). Os lactentes pré-termo com peso inferior a 1.500 gramas e/ou idade gestacional inferior a 32 semanas são considerados prioridade para o acompanhamento em serviços de saúde e vigilância do desenvolvimento (MINISTÉRIO DA SAÚDE, 2015).

Além da prematuridade, outro fator de risco para o desenvolvimento infantil são as malformações congênitas. Essa patologia atinge entre 1\% e 2\% dos nascidos vivos. Destes, aproximadamente $10 \%$ possuem deformidades dos membros superiores (KOZIN, 2003; CHUNG, 2011). Algumas dessas deformidades ocorrem de forma isolada, contudo há associações com síndromes sistêmicas que envolvem discrasias sanguíneas, cardiopatias, malformações do sistema nervoso central, malformações do tubo digestivo e atraso do desenvolvimento neuropsicomotor (KOZIN, 2004).

Os primeiros anos de vida são caracterizados por importantes aquisições das habilidades motoras (marcos motores), cognitivas e de linguagem (PAPALIA; OLDS; FELDMAN, 2006). Por se tratar de um período crítico, surge à necessidade da intervenção precoce para lactentes com fatores de risco para desenvolvimento neuropsicomotor. A intervenção precoce é caracterizada por um conjunto de atividades que tem como objetivos estimular as capacidades das crianças o mais cedo possível, apoiar as famílias e fornecer um monitoramento ativo dessas aquisições (FORMIGA; PEDRAZZANI; TUDELLA, 2010).

Entre os instrumentos de intervenção, uma das opções que vem apresentando bons resultados é a intervenção aquática (CAROMANO et al., 1998; 
TEIXEIRA-ARROYO; OLIVEIRA, 2007; TOBLE et al., 2013), pois o meio aquático oferece diminuição da sobrecarga articular e aumento dos graus de movimentos, assim como proporciona um ambiente mais agradável e lúdico (COLE; BECKER, 2004; KELLY; DARRAH, 2005). As propriedades físicas da água e os manuseios oferecidos pelo fisioterapeuta durante a abordagem dessas crianças podem facilitar ou dificultar os movimentos funcionais, o que dependerá do objetivo a ser alcançado (CAROMANO et al., 1998).

A atividade na água possibilita maior liberdade de movimentos para essas crianças, visto que a força da gravidade não é um limitador para a atividade motora. Dessa forma, a criança poderá explorar o espaço. Além disso, a atividade na água é lúdica, o que torna a terapia mais estimulante e prazerosa.

Este estudo teve como finalidade verificar a influência da fisioterapia aquática no desenvolvimento motor grosso de um lactente prematuro com diagnóstico clínico de malformação dos ossos do carpo bilateral.

\section{MÉTODO}

Trata-se de um relato de caso desenvolvido no setor de fisioterapia aquática da Associação de Assistência à Criança Deficiente (AACD) - unidade Ibirapuera, obedecendo aos princípios éticos para pesquisa envolvendo seres humanos e devidamente analisado e aprovado pelo Comitê de Ética e Pesquisa da AACD, com Parecer n $n^{\circ}$ 3.668.270.

A amostra foi constituída de um paciente do sexo masculino, nascido de parto cesárea, pré-termo (31 semanas), com peso ao nascer de 1.770 gramas, $43 \mathrm{~cm}$ de comprimento, perímetro cefálico de $30 \mathrm{~cm}$, Apgar 07 no primeiro minuto e 09 no quinto minuto. Constataram-se as seguintes intercorrências clínicas: permanência do paciente por 23 dias na unidade de terapia intensiva, síndrome do desconforto respiratório neonatal, sepse precoce, apneia, infecção neonatal e neutropenia.

O lactente apresentava diagnóstico de malformação dos ossos do carpo bilateral, confirmado por meio de dados coletados no prontuário clínico. Ele estava com 2 meses e 23 dias de idade corrigida, ou seja, 5 meses e 2 dias de idade cronológica. Entre as características, observaram-se pés calcâneos valgos, frouxidão ligamentar, hipotonia apendicular leve, desvio ulnar dos punhos e atraso no desenvolvimento neuropsicomotor. Foi inserido no programa de reabilitação da instituição. Durante o período de intervenção do estudo, o paciente realizava apenas a fisioterapia aquática e, depois de liberado pelo médico da instituição, aguardava o início das demais terapias. 


\section{PROCEDIMENTOS}

O responsável pelo paciente foi devidamente informado sobre a pesquisa. Após concordar em participar do estudo, assinou o Termo de Consentimento Livre e Esclarecido.

O paciente foi avaliado no início e no final do protocolo de intervenção de fisioterapia aquática, constituído de oito sessões de fisioterapia aquática com duração de 35 minutos cada terapia. As avaliações e intervenções foram realizadas por um único examinador.

Para verificar o desenvolvimento motor grosso desse lactente, utilizamos como instrumento de avaliação a Alberta Infant Motor Scale (Aims). Trata-se de uma escala canadense com validação para a população brasileira que avalia o desenvolvimento motor grosso de lactentes desde o nascimento (40 semanas pós-concepção) até a idade da locomoção independente (de 0 a 18 meses), por meio de 58 itens, divididos em quatro subescalas: prono (21 itens), supino (nove itens), sentado (12 itens) e de pé (16 itens). O escore total e a idade corrigida determinam a posição do lactente em uma das curvas dos percentis, desenvolvida com a amostra normativa. Para análise da Aims, serão apresentadas e classificadas as frequências de observações por faixa de percentil: desenvolvimento atípico (menor que 5\%), suspeito (5\%-25\%) e típico (mais de 25\%) (VALENTINI; SACCANI, 2012).

\section{INTERVENÇÃO}

O atendimento foi realizado uma vez por semana. Cada sessão teve a duração de 35 minutos, durante oito semanas, em uma piscina terapêutica aquecida com média de temperatura da água de $33^{\circ} \mathrm{C}$.

A intervenção foi centrada em um protocolo com enfoque no estímulo do desenvolvimento motor grosso, constituído de manuseios para o estímulo do controle de cabeça, do rolar e sentar com apoio (Quadro 1).

Quadro 1 Manuseios realizados na intervenção da fisioterapia aquática

\begin{tabular}{llll}
\hline $\begin{array}{c}\text { Posição inicial } \\
\text { do lactente }\end{array}$ & $\begin{array}{c}\text { Equipamentos } \\
\text { utilizados }\end{array}$ & $\begin{array}{c}\text { Posicionamento do terapeuta } \\
\text { e descrição do manuseio }\end{array}$ & $\begin{array}{c}\text { Objetivo do } \\
\text { exercício }\end{array}$ \\
\hline Lactente em & $\begin{array}{l}\text { No colo da terapeuta, por meio } \\
\text { de movimentação lenta, além de } \\
\text { supino }\end{array}$ & $\begin{array}{l}\text { Adaptar o lactente ao } \\
\text { meio aquático e } \\
\text { a terapeuta }\end{array}$ \\
\hline
\end{tabular}


Quadro 1 Manuseios realizados na intervenção da fisioterapia aquática (continuação)

\begin{tabular}{|c|c|c|c|}
\hline $\begin{array}{l}\text { Posição inicial } \\
\text { do lactente }\end{array}$ & $\begin{array}{l}\text { Equipamentos } \\
\text { utilizados }\end{array}$ & $\begin{array}{l}\text { Posicionamento do terapeuta } \\
\text { e descrição do manuseio }\end{array}$ & $\begin{array}{l}\text { Objetivo do } \\
\text { exercício }\end{array}$ \\
\hline $\begin{array}{l}\text { Lactente em } \\
\text { supino }\end{array}$ & & $\begin{array}{l}\text { Terapeuta com apoio inicialmente } \\
\text { em cabeça, posteriormente } \\
\text { evoluindo para apoio na região } \\
\text { axilar do lactente. }\end{array}$ & $\begin{array}{l}\text { Ativar a musculatura } \\
\text { flexora cervical. }\end{array}$ \\
\hline $\begin{array}{l}\text { Lactente em } \\
\text { prono }\end{array}$ & & $\begin{array}{l}\text { Terapeuta com apoio na região } \\
\text { axilar do lactente, assegurando- } \\
\text {-se do contato da boca do } \\
\text { paciente com a água. }\end{array}$ & $\begin{array}{l}\text { Ativar a musculatura } \\
\text { paravertebral e } \\
\text { estimular o controle } \\
\text { cervical. }\end{array}$ \\
\hline $\begin{array}{l}\text { Lactente em } \\
\text { supino com a } \\
\text { cabeça para a } \\
\text { direita e } \\
\text { posteriormente } \\
\text { para a esquerda }\end{array}$ & & $\begin{array}{l}\text { Lactente posicionado na coxa da } \\
\text { terapeuta e a profissional com } \\
\text { apoio na região dos ombros e } \\
\text { braços do lactente, estimulando } \\
\text { os movimentos laterais ao redor } \\
\text { do eixo sagito-transversal. }\end{array}$ & $\begin{array}{l}\text { Ativar os músculos } \\
\text { flexores laterais } \\
\text { cervicais. }\end{array}$ \\
\hline $\begin{array}{l}\text { Lactente em } \\
\text { prono }\end{array}$ & & $\begin{array}{l}\text { Lactente apoiando os membros } \\
\text { superiores na coxa do terapeuta e } \\
\text { este apoiando o tronco do } \\
\text { lactente. }\end{array}$ & $\begin{array}{l}\text { Estimular o controle } \\
\text { cervical. }\end{array}$ \\
\hline $\begin{array}{l}\text { Lactente em } \\
\text { prono }\end{array}$ & $\begin{array}{l}\text { Tapete de EVA } \\
\text { e brinquedo }\end{array}$ & $\begin{array}{l}\text { Lactente realizando descarga de } \\
\text { peso em antebraços e terapeuta } \\
\text { oferecendo suporte em membros } \\
\text { superiores. Posteriormente, esse } \\
\text { suporte foi retirado e o apoio } \\
\text { ocorreu em tronco. }\end{array}$ & $\begin{array}{l}\text { Estimular o controle } \\
\text { cervical e ativar a } \\
\text { musculatura } \\
\text { extensora de tronco e } \\
\text { estabilizadora de } \\
\text { cintura escapular. }\end{array}$ \\
\hline $\begin{array}{l}\text { Lactente em } \\
\text { supino }\end{array}$ & & $\begin{array}{l}\text { Terapeuta realizando apoio } \\
\text { bilateral na região axilar do } \\
\text { lactente, estimulando o rolar no } \\
\text { eixo longitudinal. }\end{array}$ & $\begin{array}{l}\text { Estimular o rolar de } \\
\text { supino para lateral/ } \\
\text { prono e ativar a } \\
\text { musculatura oblíqua } \\
\text { de tronco. }\end{array}$ \\
\hline $\begin{array}{l}\text { Lactente em } \\
\text { supino }\end{array}$ & $\begin{array}{l}\text { Tapete de EVA } \\
\text { e brinquedo }\end{array}$ & $\begin{array}{l}\text { Progressão do treino do rolar com } \\
\text { facilitação do terapeuta } \\
\text { utilizando a superfície instável e } \\
\text { dificultando a tarefa. Apoio do } \\
\text { terapeuta na região dos quadris } \\
\text { do lactente. }\end{array}$ & $\begin{array}{l}\text { Estimular o rolar de } \\
\text { supino para lateral/ } \\
\text { prono. }\end{array}$ \\
\hline
\end{tabular}

(continua) 
Quadro 1 Manuseios realizados na intervenção da fisioterapia aquática (continuação)

\begin{tabular}{|c|c|c|c|}
\hline $\begin{array}{l}\text { Posição inicial } \\
\text { do lactente }\end{array}$ & $\begin{array}{l}\text { Equipamentos } \\
\text { utilizados }\end{array}$ & $\begin{array}{l}\text { Posicionamento do terapeuta } \\
\text { e descrição do manuseio }\end{array}$ & $\begin{array}{l}\text { Objetivo do } \\
\text { exercício }\end{array}$ \\
\hline $\begin{array}{l}\text { Lactente em } \\
\text { supino }\end{array}$ & & $\begin{array}{l}\text { Terapeuta realizando apoio na } \\
\text { região lateral de tronco do } \\
\text { lactente, auxiliando o puxar-se } \\
\text { para sentado ao redor do eixo } \\
\text { fronto-transversal. }\end{array}$ & $\begin{array}{l}\text { Ativar a musculatura } \\
\text { abdominal. }\end{array}$ \\
\hline Sentada & & $\begin{array}{l}\text { Terapeuta com apoio na região } \\
\text { dos quadris do lactente. Isso } \\
\text { ocorreu por meio da rotação no } \\
\text { eixo sagito-transversal. }\end{array}$ & $\begin{array}{l}\text { Ativar a musculatura } \\
\text { flexora de tronco. }\end{array}$ \\
\hline Sentada & $\begin{array}{l}\text { Tablado } \\
\text { imerso }\end{array}$ & $\begin{array}{l}\text { Terapeuta com apoio nos quadris } \\
\text { do lactente, (nível de } \\
\text { profundidade no processo } \\
\text { xifoide). Como forma de } \\
\text { progressão do sentar, houve a } \\
\text { diminuição do nível de } \\
\text { profundidade. }\end{array}$ & $\begin{array}{l}\text { Estimular a postura } \\
\text { sentada. }\end{array}$ \\
\hline
\end{tabular}

Fonte: Elaborado pelos autores.

\section{RESULTADOS}

A Tabela 1 demonstra os valores absolutos dos escores nas subescalas (prono, supino, sentado e em pé) na avaliação pré-intervenção e pós-intervenção, assim como os valores absolutos do escore bruto (total) em cada uma das avaliações.

Tabela 1 Escores da Aims em cada etapa da intervenção

\begin{tabular}{ccc}
\hline & Pré-intervenção & Pós-intervenção \\
\hline Escore prono & 3 & 6 \\
\hline Escore supino & 2 & 7 \\
\hline Escore sentado & 0 & 3 \\
\hline Escore em pé & 1 & 2 \\
\hline Escore bruto & 6 & 18 \\
\hline
\end{tabular}

Fonte: Elaborada pelos autores. 
Na avaliação pré-intervenção, o lactente apresentou 6 pontos no escore bruto da Aims. Após a intervenção da fisioterapia aquática, apresentou escore 18. Esses 12 pontos obtidos foram observados nas posturas prona, supina, sentada e em pé. Em prono, a habilidade motora mais evoluída que o lactente apresentava antes da intervenção era a de elevar a cabeça assimetricamente a 45; após a intervenção, realizou apoio nos antebraços com cotovelos à frente dos ombros. Em supino, observou-se que o lactente na pré-intervenção realizava rotação da cabeça em direção à linha média. Em contrapartida, depois da intervenção alcançava as mãos nos pés. Sentado, na pré-intervenção, não elevava a cabeça nem a mantinha na linha média. Após a intervenção, ao tracionar para sentar, a cabeça estava alinhada e à frente do tronco. Na postura em pé, mesmo com suporte em tronco, a criança não mantinha alinhamento entre cabeça, quadril e pés, e, após a intervenção, adquiriu o alinhamento ainda com suporte.

O Gráfico 1 demonstra que, antes da intervenção, o lactente apresentou percentil 5 na Aims, e, após a intervenção, foi observado percentil 50.

Gráfico 1 Avaliação do desenvolvimento motor grosso

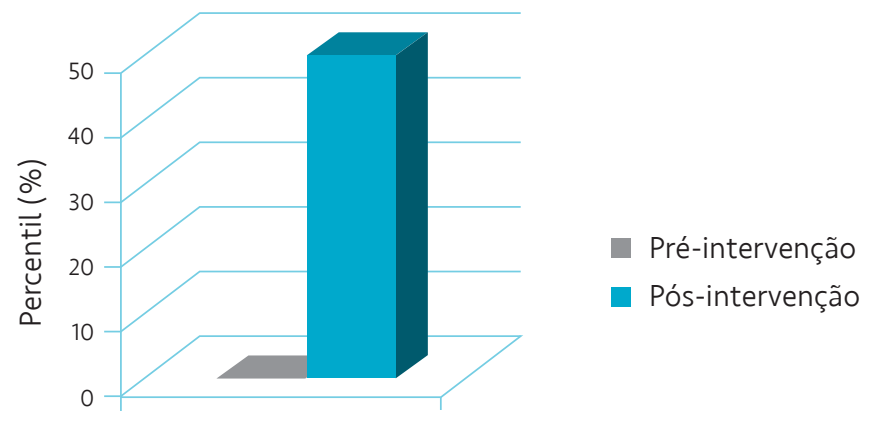

Alberta Infant Motor Scale (AIMS)

Fonte: Elaborado pelos autores.

\section{DISCUSSÃO}

Este estudo demonstrou que a fisioterapia aquática foi benéfica para o lactente prematuro com malformações congênitas de membros superiores e desenvolvimento motor grosso suspeito, visto que houve melhora da postura sentada e do controle de cabeça. 
Verificou-se, entre as aquisições observadas após a intervenção, que o lactente melhorou/adquiriu o controle de cabeça. Esse efeito corrobora os resultados obtidos por alguns autores que relataram que a ativação/fortalecimento da musculatura do pescoço é facilitada pelas propriedades de suporte do meio aquático, associadas aos manuseios, como as rotações no eixo fronto-transversal, pois esse foi um dos principais manuseios realizados neste trabalho (SILVA; BRANCO, 2011).

Os ganhos adquiridos após a intervenção têm relação direta com os princípios físicos da água, especialmente com o empuxo. Na avaliação realizada por meio da Aims, antes de iniciar a intervenção, o lactente apresentava desenvolvimento motor suspeito com percentil de $5 \%$. Esse percentil foi elevado para 50\% após a ação, considerado desenvolvimento motor típico, de acordo com a idade corrigida. No meio líquido, o empuxo tem como efeito a redução do peso corporal, tornando mais fácil a realização de movimentos de difícil execução, quando comparado ao ambiente terrestre (CARVALHO et al., 2015).

Após a intervenção, o lactente sentava com apoio dos braços e, ao tracionar para ficar sentado, a cabeça estava alinhada e à frente do tronco. Portanto, notamos melhora da força muscular de tronco e cabeça em relação ao período de pré-intervenção. Determinados autores relatam que a ativação da musculatura de tronco no meio líquido é favorecida pelo empuxo e que a viscosidade proporciona um maior tempo de respostas ao desequilíbrio, favorecendo os ajustes posturais e aprimorando o controle de tronco (SILVA; BRANCO, 2011). De acordo com os manuseios, podemos usufruir o empuxo, em conjunto com a pressão hidrostática, para a ativação de respostas automáticas de equilíbrio, estáticas ou dinâmicas, facilitando o mecanismo postural mais próximo da normalidade (CARREGARO; TOLEDO, 2008).

No presente estudo, trabalhamos com diversos níveis de profundidade, de acordo com a evolução motora observada. Esse conceito já foi descrito por outros autores, segundo os quais, à medida que o paciente evolui, pode-se diminuir a ação do empuxo por meio do nível de imersão: quando se dificulta a tarefa, o paciente é capaz de aproximar-se da realização em solo. Essa atividade que favorece o aprendizado motor é denominada transferência positiva (SILVA; BRANCO, 2011). Esse fato ficou evidente principalmente na avaliação final, quando observamos a transferência positiva.

Os estudos com fisioterapia aquática nesse grupo de pacientes com malformações congênitas de membros superiores e atraso no desenvolvimento 
neuropsicomotor são escassos. Resultados semelhantes foram encontrados em um estudo de caso, no qual se utilizou a fisioterapia aquática em um lactente com síndrome de Down e deficiência auditiva. Os autores também utilizaram a Aims como instrumento de avaliação na pré e pós-intervenção para verificar a eficácia da fisioterapia aquática na aquisição de habilidades motoras grossas e, como resultados, encontraram melhora do desempenho motor nas posturas prona e sentada (TOBLE et al., 2013).

A fisioterapia aquática pode influenciar positivamente as posturas antigravitacionais, prona e sentada por causa dos princípios físicos da água: estimulação sensorial e fortalecimento da musculatura de tronco (TOBLE et al., 2013). Além disso, proporciona experiências e vivências novas e variadas ao indivíduo e favorece a percepção sensorial e a ação motora, isto é, o desenvolvimento das capacidades psicomotoras, tais como: coordenação, equilíbrio, esquema corporal, lateralidade, orientação espacial e orientação temporal (TEIXEIRA-ARROYO; OLIVEIRA, 2007).

O lactente iniciou a intervenção aos 5 meses e 2 dias de idade cronológica, de acordo com liberação médica da AACD. A plasticidade neural fundamenta e justifica a intervenção precoce para bebês que apresentem risco potencial de atrasos no desenvolvimento neuropsicomotor. Sabe-se que a intervenção precoce contribui de forma positiva para o desenvolvimento infantil de lactentes com fatores de risco para o desenvolvimento neuropsicomotor, reduzindo sua duração e/ou gravidade, ou até mesmo minimizando as futuras sequelas, especialmente nos três ou até, no máximo, quatro primeiros meses de vida (FORMIGA; PEDRAZZANI; TUDELLA, 2010).

O estudo de Vignochi, Teixeira e Nader (2010) demonstrou a eficácia da fisioterapia aquática na redução da dor e na melhora da qualidade do sono de lactentes recém-nascidos pré-termo estáveis internados na unidade de terapia intensiva neonatal (Utin). Os autores sugeriram que a fisioterapia aquática pode ser utilizada como um método não farmacológico para aliviar a dor e melhorar a qualidade e a duração do sono profundo, contribuindo para a redução dos efeitos nocivos da Utin em lactentes, sem privá-los da estimulação tátil e cinestésica necessária para o desenvolvimento infantil. No presente estudo, a fisioterapia aquática ocorreu fora do ambiente hospitalar, isto é, em um centro de reabilitação referência na área, e com um lactente de 2 meses e 23 dias de idade corrigida. Apesar de o desfecho ter sido o desenvolvimento motor grosso do lactente, a genitora sempre relatava que, desde o início da 
intervenção, observou melhora em relação ao sono, principalmente no mesmo dia em que o filho realizava a terapia.

Pesquisadores verificaram que recém-nascidos prematuros hospitalizados apresentam redução significativa nas frequências cardíaca e respiratória (ambas dentro dos limites da normalidade) e que os seus níveis de cortisol salivar foram significativamente reduzidos após a fisioterapia aquática, sugerindo alívio em curto prazo do estresse (OLIVEIRA TOBINAGA et al., 2016). Com base nessas evidências, a fisioterapia aquática realizada com profissional qualificado pode ser introduzida como uma intervenção segura para bebês clinicamente estáveis nascidos prematuramente.

Estudos sobre a eficácia dos programas de atividade física e fisioterapia aquática na promoção do desenvolvimento motor nessa população de crianças são limitados (BLAUW-HOSPERS; HADDERS-ALGRA, 2005). Diante disso, parece que o conhecimento do que influencia os resultados do desenvolvimento neurológico é a chave para o desenvolvimento de melhores estratégias.

Foi verificado, por meio de um ensaio clínico randomizado, o efeito de programas de atividade física precoce no desempenho motor e no desenvolvimento neuromuscular de 76 bebês prematuros e internados em Utin, divididos em quatro grupos. O grupo I recebeu exercícios de amplitude de movimento passiva diária em todas as extremidades. O grupo II foi submetido diariamente à atividade física passiva realizada na água para ombros e região pélvica. $\mathrm{O}$ grupo III recebeu, em dias alternados, a combinação das atividades físicas passivas dos grupos I e II. Por fim, o grupo IV, denominado grupo contenção e composto por recém-nascidos, foi posicionado em posição fetal por 10 minutos. Ao final, observou-se que a atividade física precoce nos diferentes grupos tem os mesmos efeitos do grupo contenção no desempenho motor de recém-nascidos prematuros (VALIZADEH et al., 2017). São escassos os estudos que demonstram a interferência da fisioterapia aquática em lactentes de alto risco. Mais escassos ainda são os estudos que abordam o desenvolvimento motor nos primeiros meses de vida desses lactentes.

\section{CONCLUSÃO}

A fisioterapia aquática funcional, por meio da associação das propriedades físicas da água e dos manuseios realizados pelo terapeuta, influenciou de forma positiva o desenvolvimento motor grosso, repercutindo nas posturas prona, supina e sentada do lactente prematuro com malformação congênita dos ossos do carpo bilateral. 
Observamos que testes específicos e um acompanhamento multidisciplinar são fundamentais para lactentes de risco e, por meio deste estudo, buscamos estimular novas pesquisas relacionadas à fisioterapia aquática nessa população de risco. Para as pesquisas futuras, sugerimos a ampliação do número da amostra e a aplicação da Aims após cada sessão da intervenção.

\title{
Aquatic physiotherapy on the gross motor development of a premature infant with congenital malformations of the upper limbs: case report
}

\begin{abstract}
The congenital malformations and premature are risk factors for neuropsychomotor development. It is known that the first years of life are characterized by important acquisitions of child development. This period is considered crucial, due to greater brain plasticity, with increased neural networks and consequently developmental gains. Given these characteristics emerges the importance of intervention as early as possible. The aim of this study was to verify the influence of functional aquatic physiotherapy on the gross motor development of a premature infant with a clinical diagnosis of bilateral carpal bone malformation. This is a case report developed in the aquatic physiotherapy sector of the Assistance Association for Disabled Children (Associação de Assistência à Criança Deficiente - AACD) - Ibirapuera unit. One male patient with 5 months and 2 days of chronological age, with medical diagnosis of bilateral carpal bone malformation and neuropsychomotor developmental delay participated of this study. Evaluations were performed before and after eight sessions of intervention through the Alberta Infant Motor Scale (Aims). The protocol was defined on stimulating neuropsychomotor development focus. It was possible to observe an increase of 12 points in the total score of Aims, ranging from 6 to 18, and improvement in the percentile. Where before the intervention was verified 5 percentile, and after the intervention, 50 percentile. This study shows that for this patient functional aquatic physiotherapy can positively influence the gross motor development.
\end{abstract}

\section{Keywords}

Hydrotherapy. Child development. Prematurity. Early intervention. Congenital abnormalities 


\title{
Fisioterapia acuática en el desarrollo motor grueso de un lactante prematuro con malformaciones congénitas de la extremidad superior: reporte del caso
}

\begin{abstract}
Resumen
Las malformaciones congénitas y la prematuridad son factores de riesgo para el desarrollo neuropsicomotor. Se sabe que los primeiros años de la vida se caracterizan por importantes adquisiciones del dessarrollo infantil. Este periodo se considera crucial, debido a la mayor plasticidade cerebral, con el aumento de las redes neuronales y, en consecuencia, mejorando las ganancias del desarrollo. Dadas estas características, surge la importancia de la intervención lo antes posible. El objetivo de este estudio fue verificar la influencia de la fisioterapia acuática funcional en el desarollo motor grueso de un lactante prematuro con diagnóstico clínico de malformación del hueso del carpo (bilateral). Este en el sector de fisioterapia acuática de la Asociación para el Cuidado de Ninõs Discapacitados (Associação de Assistência à Criança Deficiente - AACD) - unidad Ibirapuera. Participó en este estudio un paciente masculino con 5 meses y 2 días de edad cronológica, con diagnóstico médico de malformación bilateral del hueso del carpo y retraso en el desarollo neuropsicomotor. Se realizaron evaluaciones, antes y despúes de ocho sesiones de atención. Además, el bebé fue evaluado a través de la Escala Motora Infantil de Alberta (Alberta Infant Motor Scale Aims). El protocolo se definió con un enfoque en estimular el desarrollo neuropsicomotor. Fue posible observar un aumento de 12 puntos en el puntaje bruto de AIMS, de 6 a 18, y uma mejora em el percentil. Antes de la intervención, se verifico el percentil 5 y, después de la intervención, el percentil 50. Este estudio demuestra que para este paciente la fisioterapia acuática funcional puede influir positivamente en el desarrollo motor grueso.
\end{abstract}

\section{Palabras clave}

Hidroterapia. Desarrollo infantil. Prematuridad. Intervención temprana. Malformaciones congénitas.

\section{REFERÊNCIAS}

BLAUW-HOSPERS, C. H.; HADDERS-ALGRA, M. A systematic review of the effects of early intervention on motor development. Developmental Medicine \& Child Neurology, v. 47, n. 6, p. 421-432, 2005. DOI: 10.1111/j.1469-8749.2005.tb01165.x 
CAROMANO, F. A. et al. Efeitos fisiológicos de sessão de hidroterapia em crianças portadoras de Distrofia Muscular de Duchenne. Revista de Fisioterapia da Universidade de São Paulo, v. 5, n. 1, p. 49-55, 1998.

CARREGARO, R. L.; TOLEDO, A. M. Efeitos fisiológicos e evidências científicas da eficácia da fisioterapia aquática. Revista Movimenta, v. 1, n. 1, p. 23-27, 2008.

CARVALHO, D. R. et al. Avaliação da capacidade funcional de exercício no ambiente aquático. Fisioterapia e Pesquisa, v. 22, n. 4, p. 355-362, 2015. DOI: 10.590/18092950/13881622042015

CHUNG, M. S. Congenital differences of the upper extremity: classification and treatment principles. Clinics in Orthopedic Surgery, v. 3, n. 3, p. 172-177, 2011. DOI: 10.4055/cios.2011.3.3.172

COLE, A.; BECKER, B. Comprehensive aquatic therapy. 2. ed. Philadelphia: Elsevier, 2004.

FORMIGA, C. K. M. R.; PEDRAZZANI, E. S.; TUDELLA, E. Intervenção precoce com bebês de risco. São Paulo: Atheneu, 2010.

KELLY, M.; DARRAH, J. Aquatic exercise for children with cerebral palsy. Developmental Medicine and Child Neurology, v. 47, n. 12, p. 838-842, 2005. DOI: 10.1017/ S0012162205001775

KOZIN, S. H. Upper-extremity congenital anomalies. The Journal of Bone \& Joint Surgery, v. 85, n. 8, p. 1564-1576, 2003. DOI: 10.2106/00004623-200308000-00021

KOZIN, S. H. Congenital disorders: classification and diagnosis. In: BERGER, R. A.; WEISS A. P. (ed.). Hand surgery. Philadelphia: Lippincott Williams \& Williams, 2004. v. 2, p. 1405-1423. DOI: 10.1016\%2FJ.JHSB.2004.02.019

MINISTÉRIO DA SAÚDE. A legislação e o marketing de produtos que interferem na amamentação: um guia para o profissional de saúde: manual técnico. Brasília: Ministério da Saúde, 2015.

OLIVEIRA TOBINAGA, W. C. de et al. Short-term effects of hydrokinesiotherapy in hospitalized preterm newborns. Rehabilitaton Research and Practice, p. 1-8, 2016. DOI: $10.1155 / 2016 / 9285056$

PAPALIA, D. E.; OLDS, S. W.; FELDMAN, R. T. Desenvolvimento humano. Porto Alegre: Artmed, 2006.

SILVA, J. B.; BRANCO, F. R. Fisioterapia aquática funcional. São Paulo: Artes Médicas, 2011.

TEIXEIRA-ARROYO, C.; OLIVEIRA, S. R. G. Atividade aquática e a psicomotricidade de crianças com paralisia cerebral. Motriz, v. 13, n. 2, p. 97-105, 2007.

TOBLE, A. M. et al. Hidrocinesioterapia no tratamento fisioterapêutico de um lactente com síndrome de Down: estudo de caso. Fisioterapia em Movimento, v. 26, n. 1, p. 231-238, 2013. DOI: 10.1590/S0103-51502013000100025 
VALENTINI, N. C.; SACCANI, R. Brazilian validation of the Alberta Infant Motor Scale. Physical Therapy, v. 92, n. 3, p. 440-447, 2012. DOI: 10.2522/ptj.20110036

VALIZADEH, L. et al. Effect of early physical activity programs on motor performance and neuromuscular development in infants born preterm: a randomized clinical trial. Journal of Caring Sciences, v. 6, n. 1, p. 67-79, 2017. DOI: 10.15171/jcs.2017.008

VIGNOCHI, C.; TEIXEIRA, P. P.; NADER, P. P. Efeitos da fisioterapia aquática na dor e no estado de sono e vigília de recém-nascidos pré-termo estáveis internados em unidade de terapia intensiva neonatal. Revista Brasileira de Fisioterapia, v. 14, n. 3, p. 214-220, 2010.

WORLD HEALTH ORGANIZATION. Born too soon: the global action report on preterm birth. Geneva: World Health Organization, 2018. 